\title{
Nonlinear fast magnetosonic waves in solar coronal holes
}

\author{
K. Murawski ${ }^{1}$, R. Oliver ${ }^{2}$, and J. L. Ballester ${ }^{2}$ \\ 1 Department of Environmental Physics, Technical University of Lublin, ul. Nadbystrzycka 40, 20-618 Lublin, \\ Poland \\ 2 Departament de Física, Universitat de les Illes Balears, 07071 Palma de Mallorca, Spain \\ e-mail: ramon.oliver@uib.es, dfsjlb0@uib.es
}

Received 2 March 2001 / Accepted 14 June 2001

\begin{abstract}
A coronal hole is modeled as a slab of cold plasma threaded by a vertical, uniform magnetic field. A periodic driver acting at the coronal base is assumed to drive the velocity component normal to the equilibrium magnetic field. Previous works indicate that, in the linear regime, only fast mode perturbations propagate, since Alfvén waves are excluded from the model and the slow wave is absent in the cold plasma limit. However, in this work, it is shown that nonlinear terms in the magnetohydrodynamic (MHD) equations give rise to excitation of the velocity component parallel to the equilibrium $\boldsymbol{B}$, with a lower amplitude than the normal component. Another consequence of nonlinearities is the generation of higher-frequency Fourier modes, which can be detected by Fourier analyzing the velocity variations above the photosphere. The nature of the nonlinear interactions in the MHD equations determines the frequency of those modes. These interactions are quadratic in the case of the parallel component, while they are cubic in the case of the normal component. Therefore, nonlinearly excited frequencies $2 \omega_{\mathrm{d}}, 4 \omega_{\mathrm{d}}, 6 \omega_{\mathrm{d}}, \ldots$ are present in the parallel velocity, whereas frequencies $3 \omega_{\mathrm{d}}, 5 \omega_{\mathrm{d}}, 7 \omega_{\mathrm{d}}, \ldots$ are present in the normal velocity, with $\omega_{\mathrm{d}}$ the driving frequency.
\end{abstract}

Key words. Sun: corona - Sun: magnetic fields - magnetohydrodynamics

\section{Introduction}

Observations of the solar corona have revealed the high complexity of magnetic field structures (November \& Koutchmy 1996). Among these structures there are extended areas, known as coronal holes, where the magnetic field is open and from which the solar wind streams upwards (Parker 1992). Photospheric foot-point motions can lead to the excitation and propagation of magnetohydrodynamic (MHD) waves in the corona (e.g., Choudhuri et al. 1993; Berghmans \& De Bruyne 1995), so the study of wave propagation in coronal holes can be an important building block towards understanding the basic physical phenomena that occur in the solar corona, and there is also the possibility that such a study may supply information about the wave heating of the solar corona (e.g., Musielak 1992; Zirker 1993) as well as provide some insight into explaining periodic oscillations observed in the solar wind (Thomson et al. 1995).

Coronal holes are regions of low-density plasma on the Sun, with magnetic fields that open freely into interplanetary space. Since thermal emission is proportional to the density squared and essentially independent of temperature, coronal holes emit little energy in X-rays and so appear dark in contrast with surrounding closed mag-

Send offprint requests to: K. Marawski

e-mail: kamur@akropolis.pol.lublin.pl netic field regions (Parker 1992). Typical values of physical parameters in coronal holes are magnetic field strength around 5-10 G, Alfvén speed of about $2000 \mathrm{~km} \mathrm{~s}^{-1}$, number density of $10^{8} \mathrm{~cm}^{-3}$ at most, and plasma temperature around $1.5 \times 10^{6} \mathrm{~K}$ (Hara et al. 1994; Ofman et al. 1997). Coronal holes are the sources of high-speed solar wind and the Ulysses spacecraft encountered a continuous fast solar wind having a velocity of about $700 \mathrm{~km} \mathrm{~s}^{-1}$ above the polar coronal holes at a distance of $\sim 1.6 \mathrm{AU}$ (Phillips et al. 1994).

Our purpose is to present a detailed description of nonlinear wave propagation in a coronal hole modeled as a slab of uniform density threaded by a vertical, straight magnetic field. The approach is to begin with a simple model that contains the relevant physics and then to build on this model to extend it to more complex cases. The rationale for this simplicity is that nonlinear effects will be easier to identify in the most basic case. Nevertheless, as a consequence of the intrinsic nonlinearity of the MHD equations, wave propagation even in such a simple structure is complex.

Analysis and simulations of linear wave propagation in the solar corona are now fairly well developed (e.g., Oliver et al. 1993; Čadež \& Ballester 1994; Čadež et al. 1995; Cadež et al. 1996; Roberts 1991). However, the linear theory is unlikely to be directly applicable for the adequate description of large perturbations and studies of 
nonlinear MHD waves have been developed (Roberts 1985; Murawski 1991; Nakariakov \& Oraevsky 1995; Nakariakov et al. 1997; Botha et al. 2000). Problems associated with propagation of MHD waves have proved difficult to solve because of nonlinear terms in MHD equations, and even seemingly simple field geometries, such as uniform vertical magnetic field, have defied analysis and numerical simulations.

The layout of the paper is as follows. The physical model employed in the present study is described in the next section. The linear wave equation and its analytical solution are presented in Sect. 3, while model nonlinear wave equations and their numerical solutions are shown in Sect. 4. The numerical model used in the present study is briefly described in Sect. 5. Results obtained by solving the full system of nonlinear MHD equations are presented in Sect. 6. Finally, the paper concludes with a short summary of results.

\section{Physical model}

Within a coronal hole the plasma $\beta \equiv p /\left(B^{2} / 2 \mu\right)$ is of the order of $10^{-2}-10^{-3}$. Consequently, gas pressure terms can be neglected and the coronal plasma can be described by the ideal MHD equations which can be written in conservative form as

$\frac{\partial \varrho}{\partial t}+\nabla \cdot(\varrho \boldsymbol{V})=0$

$\frac{\partial(\varrho \boldsymbol{V})}{\partial t}+\nabla \cdot\left[(\varrho \boldsymbol{V}) \boldsymbol{V}+I \frac{\boldsymbol{B} \cdot \boldsymbol{B}}{2 \mu}-\frac{\boldsymbol{B} \boldsymbol{B}}{\mu}\right]=0$,

$\frac{\partial \boldsymbol{B}}{\partial t}+\nabla \cdot(\boldsymbol{V} \boldsymbol{B}-\boldsymbol{B} \boldsymbol{V})=0$

$\nabla \cdot \boldsymbol{B}=0$.

Here $I$ is the $3 \times 3$ matrix, $\boldsymbol{V} \boldsymbol{V}$ is the the $3 \times 3$ tensor, $\varrho$ stands for the gas density, $\boldsymbol{V}$ is the plasma velocity, $\boldsymbol{B}$ is the magnetic field, and $\mu$ is the magnetic permeability of the plasma.

The above equations of magnetohydrodynamics are solved in a Cartesian coordinate system in which the $x$ axis is directed horizontally along the solar surface and the $z$-axis is vertical. In this paper we study two-dimensional perturbations only, hence the $y$-coordinate becomes irrelevant $(\partial / \partial y=0)$. Additionally, we assume that the $y$ components of the flow $\left(V_{y}\right)$ and magnetic field $\left(B_{y}\right)$ are identically zero, which removes the Alfvén wave from the system. Moreover, as the cold plasma approximation removes the slow wave in the linear regime, the system (1)(4) then describes the fast magnetosonic waves nonlinearly coupled to degenerate slow wave perturbations.

A coronal hole is here modeled as a slab of plasma threaded by a vertical magnetic field. The plasma is assumed homogeneous with density $\varrho_{0}$ and static $(\boldsymbol{V}=0)$. The equilibrium can be written as

$\varrho=\varrho_{0}, \quad \boldsymbol{V}=0, \quad \boldsymbol{B}=B_{0} \hat{\boldsymbol{z}}$, where $\hat{z}$ is the unit vector along the $z$-direction and $B_{0}$ is the magnetic field strength of the coronal plasma. Consequently, the square of the Alfvén speed,

$V_{\mathrm{A}}^{2}=\frac{B_{0}^{2}}{\mu \varrho_{0}}$

is constant over the entire region.

\section{Linear wave equation}

In this section we present and solve the linearized form of the equation governing the propagation of fast waves. Consider small-amplitude perturbations of the equilibrium magnetic field and density. From the linearized MHD equations it follows (e.g., Oliver et al. 1993)

$\frac{\partial^{2} V_{x}}{\partial t^{2}}-V_{\mathrm{A}}^{2}\left(\frac{\partial^{2} V_{x}}{\partial x^{2}}+\frac{\partial^{2} V_{x}}{\partial z^{2}}\right)=0$

where $V_{\mathrm{A}}$ is given by expression (6) and $V_{x}$ denotes the perturbed horizontal velocity, which lies in the direction normal to the unperturbed magnetic field. In addition, the parallel velocity component $\left(V_{z}\right)$ is identically zero. Following previous analytical work (Čadež et al. 1996), the solution to this equation, which describes linear fast magnetosonic waves, can be obtained by using Fourier transformation techniques once the initial velocity

$V_{x}(x, z=0, t)=V_{0} g_{x}(x) g_{\mathrm{t}}(t)$

at the photosphere $(z=0)$ is imposed. To obtain the solution to (7) one assumes that the amplitude of perturbations does not grow with height and that they propagate upwards from the photosphere (Čadež et al. 1996), which results in an equation formally independent of the boundary condition on the vertical derivative of $V_{x}$. In this work we consider

$g_{x}(x)=\cos \left(k_{x} x\right)$

so that the function $V_{x}(x, z, t)$ follows from the expression (Čadež et al. 1996)

$$
\begin{aligned}
V_{x}(x, z, t)= & \sqrt{\frac{2}{\pi}} V_{0} \cos \left(k_{x} x\right) \\
\times & \Re\left[\int_{0}^{k_{x} V_{\mathrm{A}}} \mathrm{e}^{-\beta z} G_{\omega}(\omega) \mathrm{e}^{i \omega t} \mathrm{~d} \omega\right. \\
& \left.+\int_{k_{x} V_{\mathrm{A}}}^{+\infty} G_{\omega}(\omega) \mathrm{e}^{i(\omega t-\alpha z)} \mathrm{d} \omega\right],
\end{aligned}
$$

with $\Re[]$ the real part of the complex quantity in the square brackets and

$$
\begin{aligned}
& \alpha=\left(\frac{\omega^{2}}{V_{\mathrm{A}}^{2}}-k_{x}^{2}\right)^{1 / 2} \\
& \beta=\left(k_{x}^{2}-\frac{\omega^{2}}{V_{\mathrm{A}}^{2}}\right)^{1 / 2}
\end{aligned}
$$


The function $G_{\omega}(\omega)$, the Fourier transform in time of the function $g_{\mathrm{t}}(t)$, is given by

$G_{\omega}(\omega)=\frac{1}{\sqrt{2 \pi}} \int_{-\infty}^{+\infty} g_{\mathrm{t}}(t) \mathrm{e}^{-i \omega t} \mathrm{~d} t$.

At this point one should mention that the choice of $g_{x}(x)$ in Eq. (9) implies that Eq. (7) for $V_{x}$ reduces to the KleinGordon equation (Čadež et al. 1996)

$\frac{\partial^{2} V_{x}}{\partial t^{2}}-V_{\mathrm{A}}^{2} \frac{\partial^{2} V_{x}}{\partial x^{2}}+\omega_{\mathrm{c}}^{2} V_{x}=0$

where the cut-off frequency $\omega_{\mathrm{c}}$ is defined as

$\omega_{\mathrm{c}}=V_{\mathrm{A}} k_{x}$.

The dispersion relation of Eq. (14),

$\omega^{2}=V_{\mathrm{A}}^{2} k_{z}^{2}+\omega_{\mathrm{c}}^{2}$,

indicates that the medium is dispersive. The lowest frequency with which fast waves can propagate upwards is equal to $\omega_{\mathrm{c}}$, so the horizontal scale introduced by the inhomogeneity in Eq. (9) leads to the appearance of dispersion in the propagation of the fast mode. Below this frequency, disturbances are evanescent and cannot carry energy high into the corona, which implies that the existence of the cut-off in the wave frequency filters out low frequencies and therefore acts as a window for oscillations.

In this work we deal with perturbations driven at the photospheric level by a photospheric driver. In the linear regime, perturbations will be detectable high in the corona as long as the driving frequency lies above the cut-off $\omega_{\mathrm{c}}$ (this has been illustrated by Čadež et al. 1996). On the other hand, if a pulse with a $\delta$-function shape is launched at $z=0$, a wave front propagates into the corona, leaving behind a wake that oscillates with frequency $\omega_{\mathrm{c}}$ (Rae \& Roberts 1982). The reason for this behavior must be found in the way the dispersion of the different modes making up the initial disturbance takes place. Each of these modes travels in the vertical direction with its own group velocity which, with the help of the dispersion relation (16), can be cast as

$V_{\mathrm{g}} \equiv \frac{\partial \omega}{\partial k_{z}}=V_{\mathrm{A}} \frac{k_{z}}{\sqrt{k_{z}^{2}+k_{x}^{2}}}=V_{\mathrm{A}} \frac{\sqrt{\omega^{2}-\omega_{\mathrm{c}}^{2}}}{\omega}$.

Formally, dispersion is defined as

$D \equiv \frac{\partial^{2} \omega}{\partial k_{z}^{2}}=V_{\mathrm{A}} \frac{k_{x}^{2}}{\left(k_{z}^{2}+k_{x}^{2}\right)^{3 / 2}}=V_{\mathrm{A}}^{2} \frac{\omega_{\mathrm{c}}^{2}}{\omega^{3}}$.

$D$ reaches the maximum value $V_{\mathrm{A}} / k_{x}^{3}$ for $k_{z}=0, \omega=\omega_{\mathrm{c}}$ and as $k_{z}$ and $\omega$ increase from these values, $D$ decreases towards zero. Hence, small frequency, long waves undergo high dispersion but possess a very small group speed, so they do not propagate too far away from the photosphere. On the contrary, large frequency, short waves suffer from little dispersion but, owing to their large group velocity, move quickly up into the corona.
The particular photospheric driver considered in this work is one starting to operate at $t=0$ and producing periodic oscillations for $t>0$. This corresponds to the function $g_{\mathrm{t}}(t)$ such that

$g_{\mathrm{t}}(t)=H(t) \sin \left(\omega_{\mathrm{d}} t\right)$,

with $H(t)$ the Heaviside function. Then, Eq. (10) reduces to

$$
\begin{aligned}
V_{x}(x, z, t)= & \frac{1}{2} V_{0} \cos \left(k_{x} x\right) \gamma(z, t)+\frac{\omega_{\mathrm{d}}}{\pi} V_{0} \cos \left(k_{x} x\right) \\
\times & \Re\left[\int_{0}^{k_{x} V_{\mathrm{A}}} \mathrm{e}^{-\beta z} G_{\omega}(\omega) \mathrm{e}^{i \omega t} \mathrm{~d} \omega\right. \\
& \left.+\int_{k_{x} V_{\mathrm{A}}}^{+\infty} G_{\omega}(\omega) \mathrm{e}^{i(\omega t-\alpha z)} \mathrm{d} \omega\right]
\end{aligned}
$$

where the function $\gamma(z, t)$ is given by

$\gamma(z, t)= \begin{cases}\mathrm{e}^{-\beta_{0} z} \sin \left(\omega_{\mathrm{d}} t\right), & k_{x}>\omega_{\mathrm{d}} / V_{\mathrm{A}} \\ \sin \left(\omega_{\mathrm{d}} t-\alpha_{0} z\right), & k_{x}<\omega_{\mathrm{d}} / V_{\mathrm{A}}\end{cases}$

with

$\alpha_{0}=\left(\frac{\omega_{\mathrm{d}}^{2}}{V_{\mathrm{A}}^{2}}-k_{x}^{2}\right)^{1 / 2}$

$\beta_{0}=\left(k_{x}^{2}-\frac{\omega_{\mathrm{d}}^{2}}{V_{\mathrm{A}}^{2}}\right)^{1 / 2}$.

\section{Properties of solutions with a homogeneous photospheric driver}

Before solving the full nonlinear set of Eqs. (1)-(4) we consider a simplified version of the problem which provides us with some insight into the more complicated twodimensional wave propagation phenomena discussed later. The dependence of all variables non $x$ is neglected and a homogeneous driver is assumed (i.e., Eq. (9) with $k_{x}=0$ ). Our purpose here is to assess the significance of nonlinear terms in wave propagation phenomena. It turns out that the MHD equations can be significantly simplified, leading to more tractable problems.

\subsection{Visual inspection of nonlinear equations}

Let us expand all plasma quantities around the equilibrium state given by Eq. (5). That is

$\varrho=\varrho_{0}+\delta \varrho$,

$V_{x}=\delta V_{x}$

$V_{z}=\delta V_{z}$

$B_{x}=\delta B_{x}$

$B_{z}=B_{0}+\delta B_{z}$,

where $\delta$ denotes perturbed quantities. After substitution of the above expansion into the MHD system (1)-(4) and 
dropping $\delta$ for simplicity, we obtain (see also Nakariakov et al. 1998)

$\frac{\partial \varrho}{\partial t}+\varrho_{0} \frac{\partial V_{z}}{\partial z}=-\frac{\partial\left(\varrho V_{z}\right)}{\partial z}$,

$\varrho_{0} \frac{\partial V_{x}}{\partial t}-\frac{B_{0}}{\mu} \frac{\partial B_{x}}{\partial z}=-\varrho \frac{\partial V_{x}}{\partial t}-\left(\varrho_{0}+\varrho\right) V_{z} \frac{\partial V_{x}}{\partial z}$,

$\varrho_{0} \frac{\partial V_{z}}{\partial t}=-\varrho \frac{\partial V_{z}}{\partial t}-\left(\varrho_{0}+\varrho\right) V_{z} \frac{\partial V_{z}}{\partial z}-\frac{1}{\mu} B_{x} \frac{\partial B_{x}}{\partial z}$,

$\frac{\partial B_{x}}{\partial t}-B_{0} \frac{\partial V_{x}}{\partial z}=-\frac{\partial\left(V_{z} B_{x}\right)}{\partial z}$

$B_{z}=0$.

Note that all linear terms appear on the left-hand side of these equations, while the right-hand sides contain the nonlinear terms.

To gain qualitative understanding of the various nonlinear interactions in the system of Eqs. (29)-(33) it is worth carrying out the following experiment. We start with the system in static equilibrium and at $t=0$ we generate a perturbation in the normal flow, $V_{x}$. The linear interaction between $V_{x}$ and $B_{x}$, Eqs. (30) and (32), gives rise to non-zero $B_{x}$ and to the propagation of these two quantities away from the photosphere, giving rise to variations of $V_{x}$ and $B_{x}$ with $z$. In the linear regime nothing else happens, so the $z$-component of the velocity and the density can only be excited nonlinearly. The first nonlinear interactions come through the last term in Eq. (31), by which $V_{z}$ is excited; this automatically gives rise to perturbations in the plasma density $\varrho$ via linear interaction with $V_{z}$. We see that, after some time, all plasma quantities (except $B_{z}$, which remains unperturbed) become nonzero.

Additional information on the evolution of the various physical parameters comes from the order of nonlinear terms. The one responsible for the excitation of $V_{z}$, $\frac{1}{\mu} B_{x} \frac{\partial B_{x}}{\partial z}$, is quadratic in the linearly perturbed quantity $B_{x}$. The flow $V_{z}$ then interacts with the density, giving rise to self-interaction of $V_{z}$ with itself. This self-interaction, described by the first two terms on the right-hand side of Eq. (31), is thus a fourth-order one. This process can be carried on to show that nonlinear terms in the equations for $\varrho$ and $V_{z}$ are of even order.

Once the parallel velocity is different from zero, the right-hand sides of Eqs. (30) and (32) contain terms of third order, fifth order, and so on, which means that the normal component of $\boldsymbol{V}$ and $\boldsymbol{B}$ are nonlinearly excited by odd order nonlinear terms. The absence of quadratic nonlinear terms in the equation for $V_{x}$ is a consequence of the lack of self-interaction of the fast wave at the second order.

\subsection{Model wave equations for $V_{x}$ and $V_{z}$}

As Eqs. (29)-(33) are still too complex for analytical treatment, we now derive a model wave equation governing wave propagation in the limit of long wavelength. To do so, we introduce a moving coordinate frame which fol- lows (with the speed of the linear wave, $V_{\mathrm{A}}$ ) the vertically propagating nonlinear disturbances. In this frame, plasma quantities depend on the spatial coordinate $z$, which means that nonlinear waves are not strictly stationary in this frame. So, we perform the following coordinate stretching (Infeld \& Rowlands 1992)

$\tau=\varepsilon \int\left(\frac{\mathrm{d} z}{V_{\mathrm{A}}}-s \mathrm{~d} t\right), \quad \xi=\varepsilon^{2} z$,

where $\varepsilon$ measures the weakness of nonlinearity and $s=$ $1(s=-1)$ corresponds to propagating waves mouving upward (down-ward). In the development that follows we keep $s$ undetermined, although we will later take $s=1$, for upward propagation. Next, we expand the perturbed plasma quantities in powers of $\varepsilon^{1 / 2}$,

$f(\xi, \tau)=f_{0}+\varepsilon^{1 / 2} f_{1}(\xi, \tau)+\varepsilon f_{2}(\xi, \tau)+\varepsilon^{3 / 2} f_{3}(\xi, \tau)+\ldots$

Since we will later retain the lower order terms only, this expansion means that we consider weakly nonlinear waves. Therefore, our approach consists of a first order improvement over the linear theory and can be incorrect for strongly (large amplitude) nonlinear waves.

Substituting expressions (34) and (35) into Eqs. (1)(4) and collecting terms at $\varepsilon^{3 / 2}$ and $\varepsilon^{2}$, we obtain after some algebra

$\varrho_{i}=s \frac{\varrho_{0}}{V_{\mathrm{A}}} V_{z i}$

$B_{x i}=-s \frac{B_{0}}{V_{\mathrm{A}}} V_{x i}$

$B_{z i}=0$,

with $i=1,2$. Additionally, we get

$\varrho_{1}=V_{z 1}=0$,

$V_{z 2}=\frac{s}{2 V_{\mathrm{A}}} V_{x 1}^{2}$.

Equations (36) and (37) confirm that $\varrho$ and $V_{z}$, on the one hand, and $V_{x}$ and $B_{x}$, on the other, suffer the same order of nonlinearity. Moreover, Eqs. (39) and (40) points out the different parity of nonlinear terms affecting those variables. Finally, Eq. (38), actually valid for $i=1,2, \ldots$, leads to Eq. (33).

A compatibility condition for the $x$-component of the momentum and induction equations at $\varepsilon^{5 / 2}$ leads to the following equation with a cubic nonlinear term,

$\frac{\partial V_{x 1}}{\partial \xi}+\frac{3}{4 V_{\mathrm{A}}^{3}} V_{x 1}^{2} \frac{\partial V_{x 1}}{\partial \tau}=0$.

Transforming this equation back into the original $z, t$ coordinates and using

$V_{x} \sim \varepsilon^{1 / 2} V_{x 1}$

we obtain

$\frac{\partial V_{x}}{\partial z}+\frac{s}{V_{\mathrm{A}}}\left(1-\frac{3}{4 V_{\mathrm{A}}^{2}} V_{x}^{2}\right) \frac{\partial V_{x}}{\partial t}=0$. 
It is now convenient to introduce the following dimensionless variables

$z^{\prime}=\frac{z}{L}, \quad t^{\prime}=t \frac{V_{\mathrm{A}}}{L}, \quad V_{x}^{\prime}=\frac{V_{x}}{V_{\mathrm{A}}}$.

In these coordinates, Eq. (43) can be rewritten as follows:

$\frac{\partial V_{x}^{\prime}}{\partial z^{\prime}}+s\left(1-\frac{3}{4} V_{x}^{\prime 2}\right) \frac{\partial V_{x}^{\prime}}{\partial t^{\prime}}=0$

from which an expression for the dimensionless wave velocity, $V_{\mathrm{w}}^{\prime}$, can be derived

$V_{\mathrm{w}}^{\prime}=\left(1-\frac{3}{4} V_{x}^{\prime 2}\right)^{-1}$

As we mentioned above, we restrict the present analysis to small amplitude, nonlinear waves, such that $V_{x}^{\prime 2} \ll 1$. The last equation shows that for these values of $V_{x}^{\prime}$ the dimensionless wave speed is always greater than one and grows with the wave amplitude $V_{x}^{\prime}$. The result $V_{\mathrm{w}}^{\prime}>1$ implies that nonlinearities speed up both positive $\left(V_{x}^{\prime}>0\right)$ and negative $\left(V_{x}^{\prime}<0\right)$ amplitude waves.

From Eq. (40) it follows that the parallel $\left(V_{z}\right)$ flow is driven by a quadratic term associated with the fast wave velocity component $\left(V_{x}\right)$. Taking

$V_{z} \sim \varepsilon V_{z 2}$

we have

$V_{z}^{\prime}=\frac{s}{2} V_{x}^{\prime 2}$

The immediate consequence of this relation is that $V_{z}^{\prime}$ is of lower magnitude than $V_{x}^{\prime}$. In terms of $V_{z}^{\prime}$, Eq. (45) can be cast as follows:

$\frac{\partial V_{z}^{\prime}}{\partial z^{\prime}}+\left(s-\frac{3}{2} V_{z}^{\prime}\right) \frac{\partial V_{z}^{\prime}}{\partial t^{\prime}}=0$

It is noteworthy that, while Eq. (45) contains a cubic nonlinear term, Eq. (49) possesses a quadratic nonlinear term. This fact, already noted after the visual inspection of Eqs. (29)-(33), has direct consequences for the dependence of the wave speed on the amplitude, be it $V_{x}^{\prime}$ or $V_{z}^{\prime}$. From Eq. (46) or Eq. (49) it follows that the wave speed is

$V_{\mathrm{w}}^{\prime}=\left(s-\frac{3}{2} V_{z}^{\prime}\right)^{-1}$.

Consequently, positive and negative velocity amplitudes parallel to the equilibrium $\boldsymbol{B}$ are speeded up and slowed down, respectively, by the quadratic nonlinearity in Eq. (49). Recently, Nakariakov et al. (2000) have studied the weakly nonlinear dynamics of linearly polarized, spherical Alfvén waves in coronal holes, showing that it can be described by means of the spherical analog of the Cohen-Kulsrud-Burgers equation. In this equation, the authors have combined the effects of spherical stratification, nonlinear steepening and dissipation due to shear viscosity, and the main stages of the wave evolution, geometrical amplification, wave breaking and enhanced dissipation, are pointed out in the results. This scenario is different fom ours since no stratification or viscosity have been included in our study and, besides, our driver excites fast waves instead of Alfvén waves. In spite of these differences, a common feature can be seen in both studies, which is the nonlinear steepening of the wave (Fig. 1a).

\subsection{Numerical solution of the model wave equations}

In this part of the paper we present numerical solutions to Eqs. (45) and (49) which are rewritten here in the compact form

$\frac{\partial V}{\partial z}+\left(1+\hat{\beta}_{n} V^{n}\right) \frac{\partial V}{\partial t}=0$,

where $\hat{\beta}_{n}$ is the normalized nonlinear coefficient and $n=1,2$ correspond to Eqs. (49) and (45), respectively. Numerical solutions to this equation are obtained by use of the fast Fourier transform method.

Numerical results for Eq. (51) with $n=2$, i.e. the model equation for $V_{x}$, are displayed in Fig. 1a, which shows the signal measured at $z=0.5 \mathrm{~L}$ as a function of time, and the corresponding Fourier spectrum (Fig. 1b). The driving signal at $z=0$ is given by

$V_{x}(z=0, t)=V_{0} \sin \left(\omega_{\mathrm{d}} t\right)$.

It is shown in Fig. 1a as a broken line for comparison with the detected signal. Here, the amplitude $V_{0}=0.3 V_{\mathrm{A}}$ and driving frequency $\omega_{\mathrm{d}}=4 \pi V_{\mathrm{A}} / L$ have been chosen. This relatively large amplitude is used to enhance the influence of nonlinearities on the velocity.

Such as predicted by Eq. (46), the most obvious effect of the cubic nonlinear term in the model equation for $V_{x}$ is to speed-up both positive and negative perturbations in such a way that the propagation speed grows with the absolute value of the velocity. As a consequence of that, peaks will never catch-up with valleys and both will move quicker than nodes. Thus, the measured signal resembles saw-tooth oscillations, clearly seen in Fig. 1a. Wave breaking can occur at a later time at the leading (left) sides of both humps and valleys, contrary to what is observed in water waves breaking on a beach.

In addition, one of the conclusions from Sect. 4.1 is that the nonlinear effects in $V_{x}$ are of odd order (third, fifth, etc.). With the sinusoidal driver (52), cubic terms are equal to a sum of trigonometric functions with arguments $\omega_{\mathrm{d}}$ and $3 \omega_{\mathrm{d}}$. In the same way, fifth order terms contain periodic variations with frequencies $\omega_{\mathrm{d}}$ and $3 \omega_{\mathrm{d}}$, and $5 \omega_{\mathrm{d}}$, and so on for higher orders. Hence, it is expected that the power spectrum of the signal measured at $z=0.5 L$ shows traces of power at frequencies $(2 n+1) \omega_{\mathrm{d}}$ with $n=1,2,3,4, \ldots$ and that higher order modes possess smaller amplitudes since they contribute less to the solution because they acquire less energy. Nevertheless, they are important since they are responsible for producing the steep profiles in Fig. 1a. We have therefore estimated the power content in this time series using the Maximum Entropy Method (MEM) power spectrum estimation (Press et al. 1989). MEM spectral estimation is 

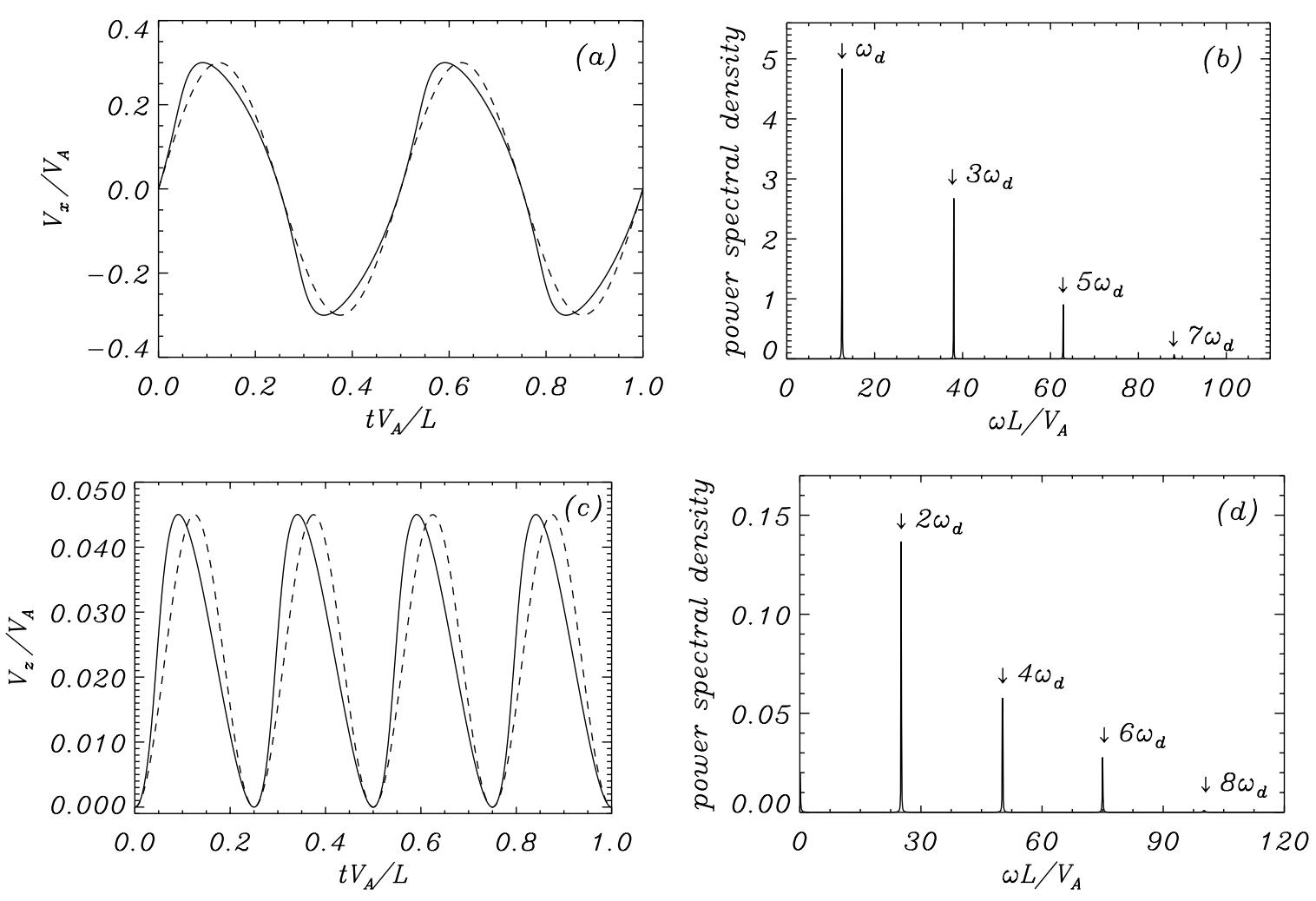

Fig. 1. a) Numerical solution to the nonlinear wave Eq. (51) with $n=2$. The solid line represents the signal detected at height $z=0.5 \mathrm{~L}$ displaced $-0.5 \mathrm{~L} / V_{\mathrm{A}}$ in time so that the disturbance is only shown from its time of arrival to the detection point. The broken line corresponds to the driving signal at $z=0$ (Eq. (52)). The driving frequency and amplitude are $\omega_{\mathrm{d}}=4 \pi V_{\mathrm{A}} / L$ and $V_{0}=0.3 V_{\mathrm{A}}$. b) MEM spectral estimation of the detected signal in a) using $410 \mathrm{MEM}$ coefficients. Peaks can be seen at frequencies $\omega_{\mathrm{d}}, 3 \omega_{\mathrm{d}}, 5 \omega_{\mathrm{d}}$, and $7 \omega_{\mathrm{d}}$, indicated by arrows. c) Same as a) with $n=1$ and driver given by Eq. (53). d) MEM spectral estimation of the detected signal in c) using 365 MEM coefficients. Peaks can be seen at frequencies $2 \omega_{\mathrm{d}}, 4 \omega_{\mathrm{d}}, 6 \omega_{\mathrm{d}}$, and $8 \omega_{\mathrm{d}}$, indicated by arrows.

sometimes better than other spectral techniques (such as FFT) at defining the positions of peaks with substantial power, although it does not predict their power well: a change in the number of MEM coefficients can drastically change the value of the estimated power, but it will not introduce spurious peaks. MEM spectral estimation for $V_{x}$ is shown in Fig. 1b. There is no doubt about the presence of power at odd multiples of the driving frequency in the signal.

The above discussion refers to $V_{x}$. Oscillations in $V_{z}$ are now excited by a driver described by

$V_{z}(z=0, t)=\frac{1}{2} V_{0}^{2} \sin ^{2}\left(\omega_{\mathrm{d}} t\right) \equiv-\frac{1}{4} V_{0}^{2}\left[\cos \left(2 \omega_{\mathrm{d}} t\right)-1\right],(53)$

where $V_{0}=0.3 V_{\mathrm{A}}$ and $\omega_{\mathrm{d}}=4 \pi V_{\mathrm{A}} / L$ have been used. The form of this driver is suggested by Eq. (48) with $s=1$. The results of solving Eq. (51) with $n=1$ are shown in Fig. 1c, where the solid and broken lines are the detected and driving velocities. As a consequence of the quadratic nonlinear term in Eq. (49), the positive signal is speeded-up and so the leading part (left part of waves in Fig. 1c) of the wave is steepened while the trailing part is smoothed out.

Now, even multiples of $\omega_{\mathrm{d}}$ are expected to shape the detected signal. This is confirmed by its power spectrum, although leakage appearing in the FFT prevents us from accurately determining the position of peaks and, therefore, we have used MEM spectral estimation which is highly sensitive to the presence of periodic components in the signal and produces narrow peaks. MEM does reveal the presence of power at the driving frequency $2 \omega_{\mathrm{d}}$ and at nonlinearly driven Fourier harmonics with frequencies $2 n \omega_{\mathrm{d}}, n=2,3,4$ (Fig. 1d). It is noteworthy that nonlinear effects on Fourier modes have also been observed by other authors (Allan et al. 1991; Botha et al. 2000; Nakariakov et al. 2000).

\section{Numerical solution of MHD equations}

The set of nonlinear MHD Eqs. (1)-(4) has been solved with a two-dimensional resistive MHD code (DeVore 1991) in which resistivity and pressure terms have been set to zero. The code utilizes the flux-corrected transport method (Boris \& Book 1973; Zalesak 1979; DeVore 1991; Murawski \& Goossens 1994b), which yields accurate results near steep gradients and moving contact discontinuities by adding numerical diffusion and antidiffusion fluxes to the scheme. Dissipation is added only in small localized regions to eliminate short-wavelength oscillations generated by numerical dispersion, while leaving the longer-wavelength phenomena minimally affected. 

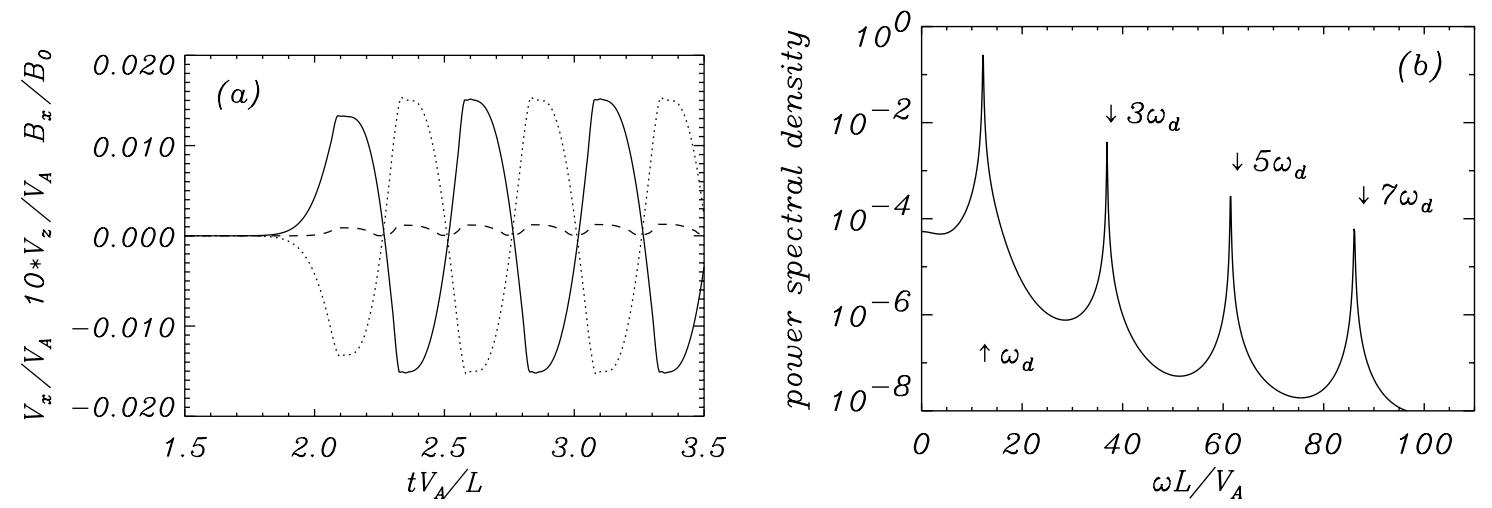

Fig. 2. Numerical solution to the nonlinear MHD Eqs. (1)-(4) for an $x$-independent driver (Eq. (54) with $n=0)$ with frequency $\omega_{\mathrm{d}}=4 \pi V_{\mathrm{A}} / L$ and amplitude $V_{0}=0.3 V_{\mathrm{A}}$. a) Time evolution of $V_{x}$ (solid line), $V_{z}$ (broken line), and $B_{x}$ (dotted line) at $x=0$, $z=2 \mathrm{~L}$. Compare $V_{x}$ and $V_{z}$ with Figs. 1a and 1c. b) MEM spectral estimation of $V_{x}$ in a) using 365 MEM coefficients. A logarithmic axis has been used for the power spectral density to enhance the presence of peaks at odd multiples of the driving frequency.

To achieve this, the dissipation has to be nonlinear and implemented locally, at regions of high-gradients. The antidiffusion fluxes are "corrected" to preserve the positivity and monotonicity of the profiles.

The resulting computer code (DeVore 1991) is fourthorder accurate in space and second-order accurate in time, and the zero divergence of the magnetic field is preserved to within machine-dependent round-off errors. An explicit predictor-corrector method has been applied for discretization in time. Modified versions of this code were successfully applied to simulate the resonant absorption (Murawski et al. 1996) and phase-mixing (Nakariakov et al. 1997) of nonlinear Alfvén waves.

\section{Numerical results}

The boundaries of the simulation box are placed at $x=$ $\pm L, z=0$, and $z=8 L$. The computational box is divided into a non-uniform grid with 100 cells in the $x$-direction and 400 cells in the $z$-direction. At $z=0$ and $z=8 L$ we use free boundary conditions with the first normal derivative at the point adjacent to the boundary set to zero. Free boundary conditions at $x= \pm L$ are employed in the case of a horizontally homogeneous driver, while rigid walls are implemented for a nonhomogeneous driver by setting $V_{x}=0$ there. These boundary conditions have been found to work well. Tests have been performed with a larger number of grid points, $300 \times 500$, and no significant differences in the solutions have been found. This fact ensures grid convergence and that the accuracy of results is not compromised by the grid size. Therefore, we conclude that the solution has been adequately resolved by the original mesh of $100 \times 400$ points.

In order to maintain high spatial resolution in particular regions, we employ a nonuniform grid in which the computational cells vary in size. The cell size changes gradually, so that neighboring cell dimensions are stretched by only a few tens of a percent per cell (typically $0.2 \%$ ). Cell sizes were set in such a way that the finest resolution is in the center of the simulation box. For this purpose the grid generation routine (GGEN) has been adapted from the ZEUS2D code (Stone \& Norman 1992).

Initially, at $t=0$, all plasma parameters are set to the homogeneous state (Eq. (5)). The system is made to evolve by specifying the flow along the lower boundary, which in our model represents the base of the coronal hole. This flow is excited by a driver described by

$V_{x}(x, z=0, t)=V_{0} \cos \left(n \frac{\pi}{2 L} x\right) \delta(z) H(t) \sin \left(\omega_{\mathrm{d}} t\right)$,

where $\delta(z)$ and $H(t)$ are the Dirac delta and Heaviside functions, respectively. The driver begins to work at $t=0$, exciting fast magnetosonic waves, and acts for the entire run. The above expression is identical to Eq. (8), with Eqs. (9) and (19), except for the explicit delta function in the $z$-direction included here and the choice $k_{x}=n \pi / 2 L$ required to satisfy the lateral boundary conditions.

In this paper, a coronal hole $200000 \mathrm{~km}$ wide (such as the one in Hara et al. 1994) is considered, which corresponds to $L=10^{5} \mathrm{~km}$. The value of the Alfvén speed is taken as $V_{\mathrm{A}}=1000 \mathrm{~km} \mathrm{~s}^{-1}$, so that the Alfvén transit time is $L / V_{\mathrm{A}}=100 \mathrm{~s}$. The driving frequency in Eq. (54) has been taken in the range $\pi V_{\mathrm{A}} / L$ to $4 \pi V_{\mathrm{A}} / L$, so the driving period varies between 50 and $200 \mathrm{~s}$. These values are similar to typical values that can be found in the literature (e.g. Parker 1992). Moreover, different heights of the detection point have been considered and we will concentrate on two of them, $200000 \mathrm{~km}$ and $400000 \mathrm{~km}$ (i.e., $2 L$ and $4 L$ ) above the photosphere. Finally, driving amplitudes in the range $0.003 V_{\mathrm{A}}-0.03 V_{\mathrm{A}}$ have been used, which in dimensional units corresponds to $3-30 \mathrm{~km} \mathrm{~s}^{-1}$.

\subsection{Homogeneous driver}

We first consider perturbations excited by a homogeneous photospheric driver, described by Eq. (54) with $n=0$. We expect the results found in Sect. 4 , in which $x$-independence was assumed, to be applicable here. 

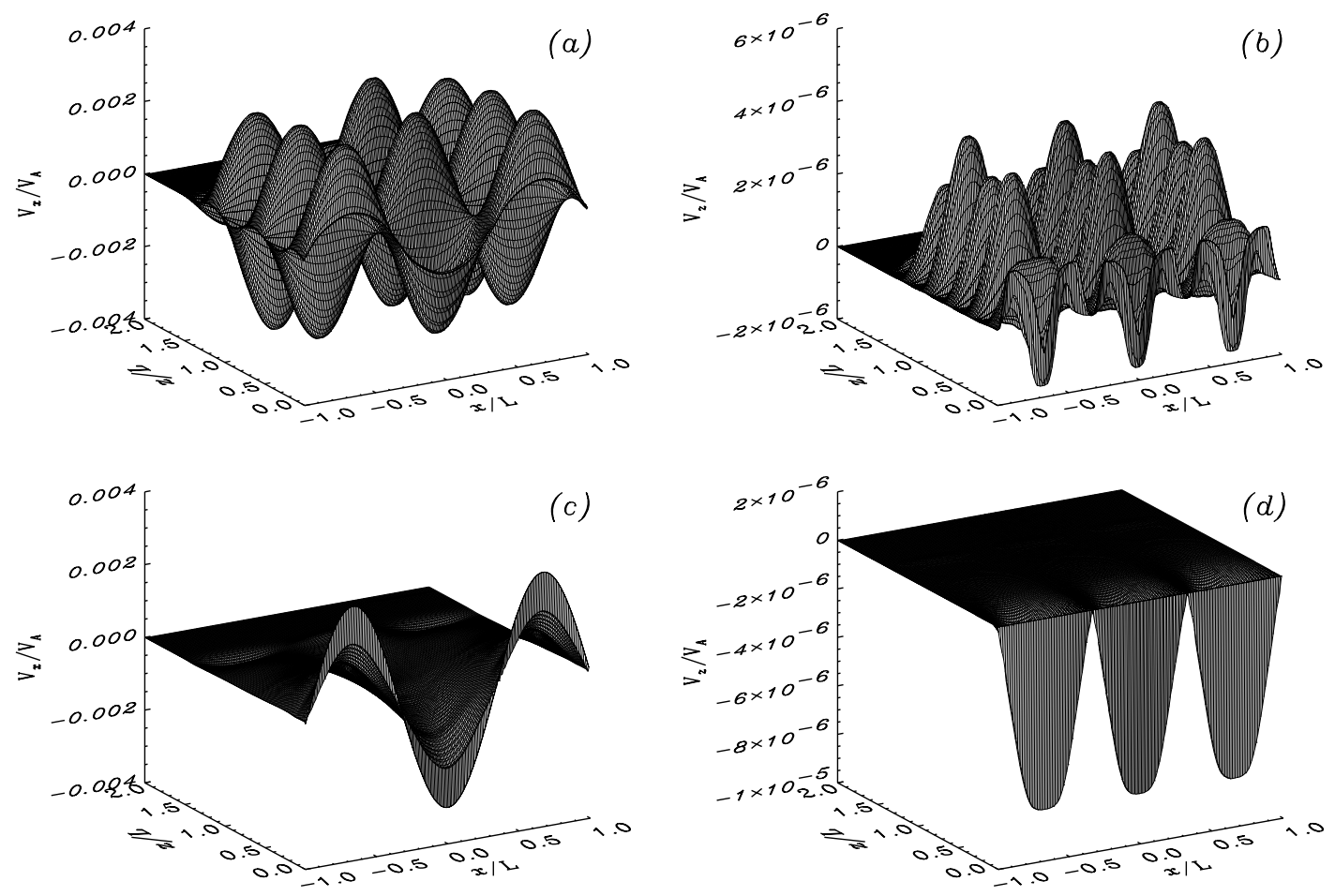

Fig. 3. Spatial distribution of a) $V_{x}$ and b) $V_{z}$ at $t=\frac{3}{2} L / V_{\mathrm{A}}$ for a driver given by Eq. (54) with $n=3, V_{0}=0.003 V_{\mathrm{A}}$, and $\omega_{\mathrm{d}}=4 \pi V_{\mathrm{A}} / L$. This driving frequency lies well above the cut-off frequency $\omega_{\mathrm{c}}$. c) and d) Same as a) and $\left.\mathbf{b}\right)$ with $\omega_{\mathrm{d}}=\pi V_{\mathrm{A}} / L$, below the cut-off frequency. Note that perturbations in $V_{x}$ and $V_{z}$ are now purely evanescent; moreover, $V_{x}$ reveals some high frequency, short wavelength waves propagating into the corona.

Numerical results for the full set of MHD equations are displayed in Fig. 2a, in which $V_{x}, V_{z}$, and $B_{x}$ are shown. The three signals have been measured at the point $(x=0$, $z=2 L)$ as a function of time and, in order to enhance nonlinear effects, $V_{0}$ is set to $0.3 V_{\mathrm{A}}$. As a consequence of the cubic nonlinearity acting on the normal flow, $V_{x}$ exhibits steepening and results in saw-tooth oscillations such as those found in the previous section (compare solid lines in Figs. 1a and 2a).

Figure $2 \mathrm{a}$ also indicates that the parallel flow, $V_{z}$, is of lower magnitude and possesses twice higher frequency than $V_{x}$. This is in agreement with the analytical finding given by formula (48). In the full set of nonlinear MHD equations, $V_{z}$ is nonlinearly driven by a quadratic term (cf. Eq. (31)) which, given the form of the driver of $V_{x}$ (Eq. (54)), is equivalent to driving $V_{z}$ by a driver with a time dependence similar to Eq. (53). Hence, it is not surprising to find such a good agreement between the solid curve in Fig. 1c and the broken curve in Fig. 2a.

Finally, comparison between the results for the horizontal component of the velocity and magnetic field shows that they oscillate in antiphase with the same amplitude. In fact, $V_{x}$ and $-B_{x}$ are visually indistinguishable. This is in agreement with the analytical procress performed before. Indeed, using $B_{x} \sim \varepsilon^{1 / 2} B_{x 1}$ and Eq. (37) with $n=1$ we obtain

$B_{x}^{\prime}=-V_{x}^{\prime}$

where $B_{x}^{\prime}=B_{x} / B_{0}$ is the dimensionless horizontal magnetic field component.
We have again computed the power spectra and MEM spectral estimate of the signals to study their power content for a range of frequencies. It is found that $V_{x}$ displays power at the driving frequency, $\omega_{\mathrm{d}}$, plus power (with decreasing amplitude) at odd multiples of $\omega_{\mathrm{d}}$ (see Fig. 2b). On the other hand, $V_{z}$ contains power at even multiples of the driving frequency (not shown). These results are also in agreement with the weakly nonlinear study performed before.

\subsection{Inhomogeneous driver}

In this subsection, results for driver (54) with $n \neq 0$ are presented. As the horizontal wavenumber is now different from zero, it follows from formula (16) that fast magnetosonic waves are dispersive in the linear regime and there is a cut-off frequency $\left(\omega_{\mathrm{c}}=n \frac{\pi}{2} V_{\mathrm{A}} / L\right)$ below which these waves are evanescent.

Let us consider in some detail the case $n=3$ for which $\omega_{\mathrm{C}}=3 \frac{\pi}{2} V_{\mathrm{A}} / L$ and let us take a driver with small amplitude, $V_{0}=0.003 V_{\mathrm{A}}$, and $\omega_{\mathrm{d}}=4 \pi V_{\mathrm{A}} / L>\omega_{\mathrm{c}}$. After three driving periods, that is, at $t=\frac{3}{2} L / V_{\mathrm{A}}$, perturbations have traveled from the photosphere up to $z=1.5 \mathrm{~L}$, showing no signs of vertical evanescence (see Figs. $3 \mathrm{a}$ and $\mathrm{b}$ ). The spatial profiles of $V_{x}$ and $V_{z}$ are consistent with Eq. (48); they reveal $\cos \left(\frac{3 \pi}{2} x\right)$ and $\cos ^{2}\left(\frac{3 \pi}{2} x\right)$ structures, respectively; in the vertical direction something similar takes place, up to the height where the system has been disturbed; and their maximum amplitudes are about $3 \times 10^{-3}$ and $4 \times 10^{-6}$. 

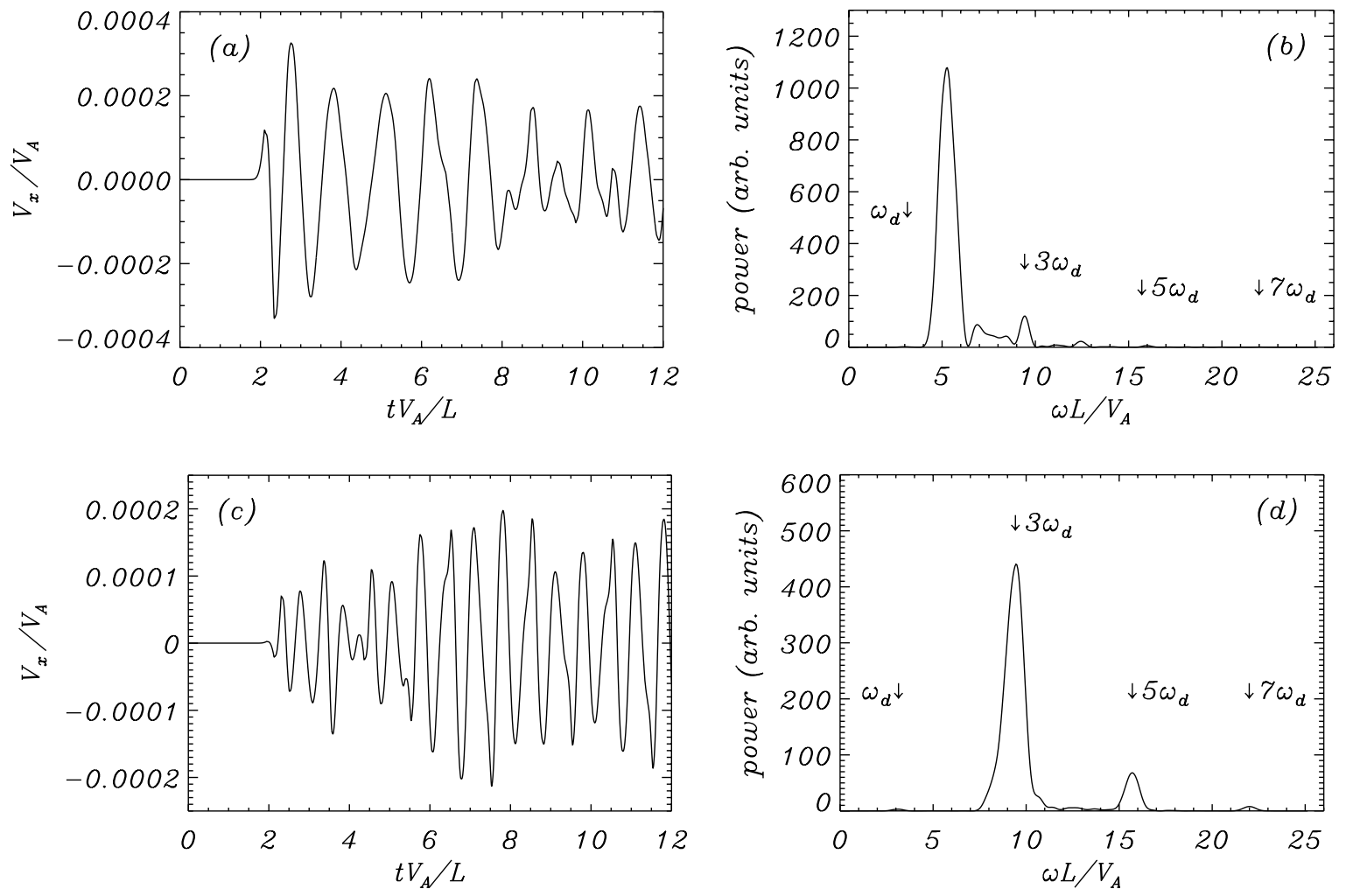

Fig. 4. a) Time evolution of $V_{x}$ at $x=0, z=2 L$ for $n=3$ and a driving amplitude $V_{0}=0.003 V_{\mathrm{A}}$ and frequency $\omega_{\mathrm{d}}=\pi$ $V_{\mathrm{A}} / L$; see Eq. (54). b) Fourier spectrum corresponding to $V_{x}$ in a), showing the existence of nonlinearly driven modes above the cut-off frequency $\omega_{\mathrm{c}}=\frac{3 \pi}{2} V_{\mathrm{A}} / L$. Power around $\omega=5 V_{\mathrm{A}} / L$ comes from the Fourier transform of the function $H(t) \sin \left(\omega_{\mathrm{d}} t\right)$; $\operatorname{see}$ text. c) Same as a) with $n=5$. Velocity oscillations seem to be of higher frequency than those in a). d) Same as b); the cut-off frequency is now $\omega_{\mathrm{c}}=\frac{5 \pi}{2} V_{\mathrm{A}} / L$, so modes below this frequency become evanescent (compare b) and $\mathbf{d}$ )). Prior to computing the power spectra the data have been multiplied by a Parzen window function (Press et al. 1989) to reduce leakage amplitude.

On the other hand, the above scenario drastically changes when the driving frequency is reduced below the cut-off, since perturbations become vertically evanescent. Figures $3 \mathrm{c}$ and d present the spatial profiles of $V_{x}$ and $V_{z}$ corresponding to $\omega_{\mathrm{d}}=\pi V_{\mathrm{A}} / L<\omega_{\mathrm{c}}$. Owing to the dispersive nature of the system in the linear regime, there are only high $\omega$, high $k_{z}$ propagating waves associated with $V_{x}$. Obviously, these modes possess frequencies above the cut-off and would not exist if the driver would be acting from $t=-\infty$. In our numerical simulation, however, it starts acting at $t=0$, so its spectrum of frequencies is not just the Fourier transform of $\sin \left(\omega_{\mathrm{d}} t\right)$, but that of $H(t) \sin \left(\omega_{\mathrm{d}} t\right)$; see Eq. (54). The Fourier transform in time of this function contains a term inversely proportional to $\omega_{\mathrm{d}}^{2}-\omega^{2}$, so frequencies other than the driving one contribute to the temporal evolution of disturbances above the photosphere.

The normal velocity component is next collected above the photosphere (at the point $x=0, z=2 L$ ) and shown in Fig. 4a. Note that perturbations with the driving frequency are evanescent, so the amplitude of $V_{x}$ is much smaller than that of the driver. The power spectrum (Fig. 4b) shows that there is no power below the cutoff frequency $\omega_{\mathrm{c}}=\frac{3 \pi}{2} V_{\mathrm{A}} / L$ and that nonlinearly driven modes appear at $\omega=3 \omega_{\mathrm{d}}$ and $\omega=5 \omega_{\mathrm{d}}$ (this peak is barely visible, but evident upon a visual analysis of the power val- ues). As these nonlinearly driven modes have frequencies above $\omega_{\mathrm{c}}$, they can freely propagate through the medium and contribute to the oscillations present in Fig. 3c. There is still another feature in Fig. $4 \mathrm{~b}$, a peak at $\omega=5 V_{\mathrm{A}} / L$, that deserves some comment. Such as discussed above, the effect of starting the simulation at a finite time, through the inclusion of the Heaviside function in Eq. (54), is to add a term proportional to $\left(\omega_{\mathrm{d}}^{2}-\omega^{2}\right)^{-1}$ to the Fourier spectrum of perturbations. It is the power coming from the part of this term above cut-off that produces the peak at $\omega=5 V_{\mathrm{A}} / L$, which has nothing to do with dispersion, nor with nonlinear effects. To prove this point we have increased the cut-off frequency by selecting a driver with $n=5$ (Fig. 4c). The Fourier spectrum of the collected signal (Fig. 4d) has no sign of that peak, which corresponds to evanescent modes now. In this figure some power at $\omega_{\mathrm{d}}$ (coming from linearly driven evanescent modes whose amplitude has not yet gone to zero at $z=2 L$ ) and $7 \omega_{\mathrm{d}}$ (coming from nonlinear driving) can be also observed.

Figure 5, made for the larger amplitude $V_{0}=0.03 V_{\mathrm{A}}$, allows us to check the effect of nonlinearities on the time signature and the corresponding power spectrum. In this case the amplitude of nonlinearly driven modes above the cut-off frequency is enhanced in comparison to the case of lower amplitude driver; compare Fig. 4b and Fig. 5b. 

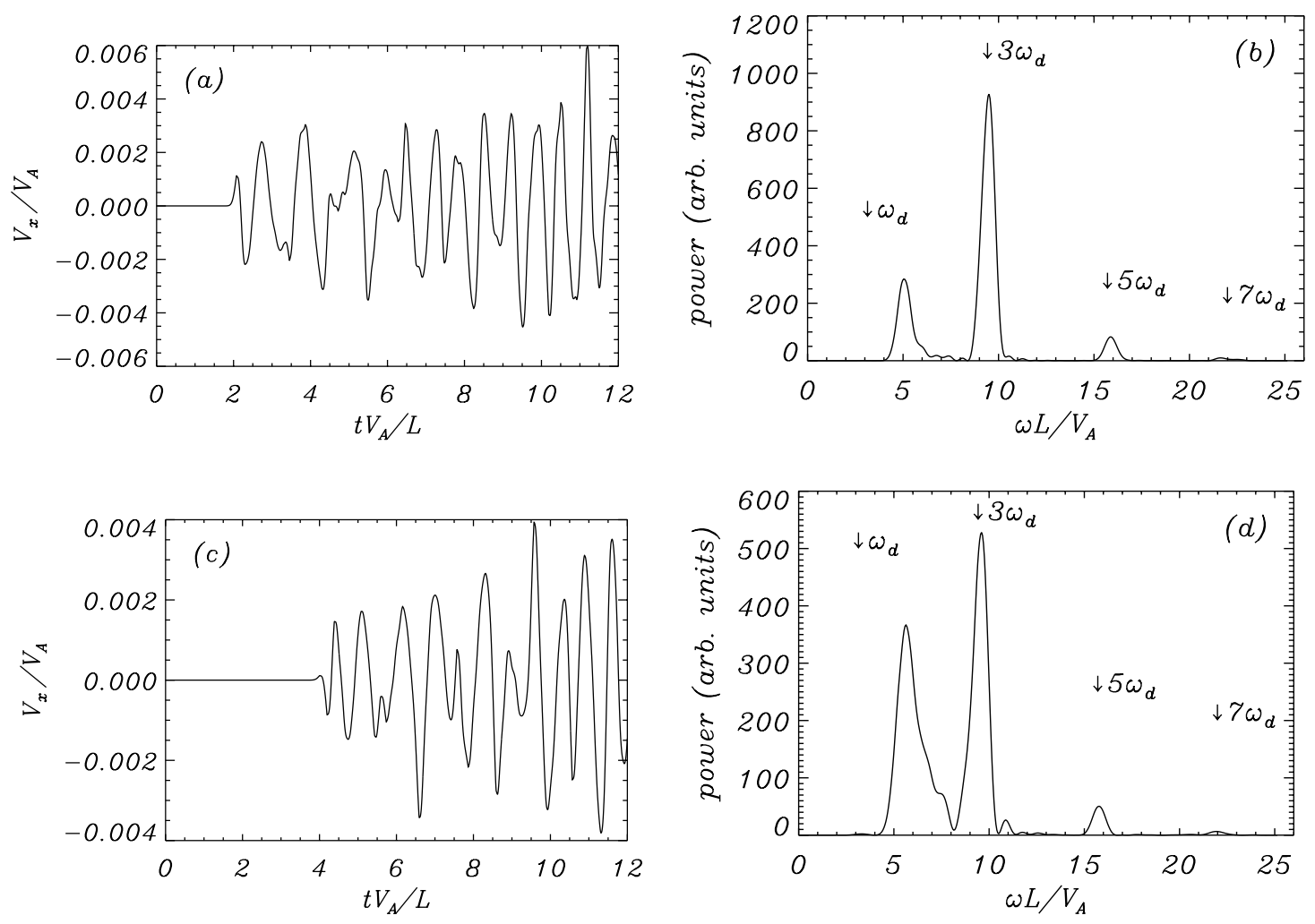

Fig. 5. a) Temporal evolution of $V_{x}$ at $x=0, z=2 L$ for a driver with $\omega_{\mathrm{d}}=\pi V_{\mathrm{A}} / L, V_{0}=0.03 V_{\mathrm{A}}$, and $n=3$. b) Power spectrum of $V_{x}$ in a); because of the larger driving amplitude, nonlinear effects are more important now (compare with Fig. 4b). c) Same as a) at $x=0, z=4 L$. d) Power spectrum of $V_{x}$ in c). Prior to computing the power spectra the data have been multiplied by a Parzen window function (Press et al. 1989) to reduce leakage amplitude.

Once the main nonlinear effects of the fast mode have been investigated, it is of interest to consider the influence of the detection point height above the photosphere. The horizontal velocity component at $z=2 L$ and $z=4$ $L$ are shown in Figs. 5a and c, respectively. Their power spectra (Figs. 5b and d) look very similar and show the now familiar peaks at 3,5 , and 7 times the driving frequency, together with the peak coming from the power generated just above the cut-off frequency in the linear regime $\left(\omega L / V_{\mathrm{A}} \simeq 5\right)$. This peak maintains the same power in the two spectra, but the other three appear to contain more power in the lower detection point. Nevertheless, the two signals have been recorded from $t=0$ to $t V_{\mathrm{A}} / L=12$, so the one in Fig. 5a contains 10 time units of useful data, while the one in Fig. 5c contains only 8 time units of useful data. This difference causes the decrease in the power of the peaks at $3 \omega_{\mathrm{d}}, 5 \omega_{\mathrm{d}}$, and $7 \omega_{\mathrm{d}}$, which can be proved by considering the time span $0<t V_{\mathrm{A}} / L<10$ at the low detection point before performing the spectral analysis. Then, the heights of peaks in the power spectrum become similar to those in Fig. 5d, which allows us to conclude that in the present circumstances nonlinearities show no dependence with height.

In this subsection we have made reference to the normal velocity component only. The analysis of the other physical quantities confirms our findings of Sect. 4, namely that the density and vertical magnetic field component change very little from their equilibrium values during the computations; the horizontal magnetic field component remains almost equal to $-V_{x}$, as predicted by Eq. (55); and the parallel velocity component is nonlinearly driven by the normal component and its power spectrum reveals the existence of power at even multiples of the driving frequency. The effects on $V_{z}$, which always remains small compared to $V_{x}$, are more evident as the driving amplitude is increased to go from the linear to the nonlinear regime.

\section{Summary}

Using a simple magnetically inactive solar coronal hole model, namely, a two-dimensional Cartesian box with uniform vertical magnetic field, constant mass density, no gravity, and no gas pressure effects, we have investigated nonlinear fast magnetosonic waves which are excited at the foot-points of magnetic field lines by a periodic driver with a specific frequency and horizontal distribution. The slow and Alfvén modes have been eliminated by considering the low plasma- $\beta$ limit and by taking $V_{y}=B_{y}=0$, $\partial / \partial y=0$, respectively.

Our model is complementary to recent numerical simulations (Murawski \& Goossens 1994a; Murawski et al. 1996; Nakariakov et al. 1997; Nakariakov et al. 2000; Botha et al. 2000). We have benefited from these 
studies to gain an improved physical insight into the process of nonlinear wave propagation in straight magnetic field lines. In particular, we have learned that time signatures of the normal and parallel velocity components differ from those in the linear case, revealing that nonlinear effects give rise to new Fourier modes in these signatures.

The investigation of nonlinear effects has been done in four steps and previous results, derived in the linear approximation (Čadež et al. 1996), have been used. The first step is rather qualitative and consists of the visual inspection of the MHD equations supplemented by a simple "thought experiment" in which the time evolution of the physical variables is followed. This experiment indicates that, when the horizontal velocity component is driven, $V_{x}$ and $B_{x}$ are affected by cubic nonlinear interactions, while $V_{z}$ and $\varrho$ are affected by quadratic nonlinear effects. In addition, $B_{z}$ does not change in time.

The second step is the derivation and solution of model equations for two representative plasma variables, the normal and parallel velocity components, for a driver with no horizontal structure. It is not necessary to bother about $B_{x}$ and $\varrho$, since they are closely related to $V_{x}$ and $V_{z}$, respectively. The two model equations have been solved numerically and the following picture of nonlinear interactions has emerged. The shape of $V_{x}$ is not sinusoidal, as in the linear regime, but rather is saw-tooth (see also Nakariakov et al. 2000). This distortion comes from the nonlinear excitation of odd multiples of the driving frequency, such as predicted by the cubic nature of nonlinearities affecting $V_{x}$. Moreover, $V_{z}$ is driven by quadratic nonlinear interactions and so its power spectrum is dominated by even multiples of the driving frequency.

The third step, the numerical solution of the full system of MHD equations with a uniform (in $x$ ) driver, has allowed us to perform a direct comparison with the results coming from the model equations and an excellent agreement has been found. Finally, the photospheric structure of the driver has been included in the fourth step. Its main influence is to introduce a cut-off frequency below which modes become evanescent. This feature is a linear one that, in conjunction with the nonlinear picture in the case of a uniform driver, results in nonlinearly driven modes becoming evanescent when their frequencies lie below the cut-off.

Future work should improve on the very simple equilibrium model used here. First of all, one could include vertical stratification of the plasma as a consequence of gravity. Then, a more sophisticated equilibrium configuration could be used in which the expansion of the coronal hole plasma to form the solar wind is added.

Furthermore, the effect of the plasma- $\beta$ in the solutions should also be investigated.

Acknowledgements. The authors express their thanks to Dr. T. D. Arber for his stimulating comments. K. M. wishes to acknowledge the financial support received from the State Committee for Scientific Research in Poland, KBN grant no. 2 PO3D 01717 and the Universitat de les Illes Balears.
He expresses his thanks to Dr. Rick DeVore for providing the original code. R. O. and J. L. B. want to acknowledge the financial support received from DGICYT and MCyT under grants PB96-0092 and BFM2000-1329 This work was performed in the framework of NATO grant no. PST.CLG.976135.

\section{References}

Allan, W., Manuel, J. R., \& Poulter, E. M. 1991, J. Geophys. Res., 96, 11461

Berghmans, D. \& De Bruyne, P. 1995, ApJ, 453, 495

Boris, J. P., \& Book, D. L. 1973, J. Comp. Phys., 11, 38

Botha, G. J. J., Arber, T. D., Nakariakov, V. M., \& Keenan, F. P. 2000, A\&A, 363, 1186

Čadež, V. M., \& Ballester, J. L. 1994, A\&A, 292, 669

Čadež, V. M., Oliver, R., \& Ballester, J. L. 1995, Solar Phys., 159,229

Čadež, V. M., Oliver, R., \& Ballester, J. L. 1996, A\&A, 314, 636

Choudhuri, A. R., Auffret, H., \& Priest, E. R. 1993, Solar Phys., 143, 49

DeVore, C. R. 1991, J. Comp. Phys., 92, 142

Hara, H., Tsuneta, S., Acton, L. W., Lemen, J. R., \& Ogawara, Y. 1991, in X-ray solar physics from Yohkoh, ed. Y. Uchida, T. Watanabe, K. Shibata, \& H. S. Hudson (Universal Acad. Press), 217

Infeld, E., \& Rowlands, G. 1992, Nonlinear waves, solitons and chaos (Cambridge, Cambridge Univ. Press), 99

Murawski, K. 1991, Acta Phys. Polon., 80, 495

Murawski, K., \& Goossens, M. 1994a, A\&A, 286, 952

Murawski, K., \& Goossens, M. 1994b, Solar Phys., 145, 65

Murawski, K., DeVore, C. R., Parhi, S., \& Goossens, M. 1996, Planet. Space Sci., 44, 253

Musielak, Z. E. 1992, Mem. Soc. Astron. It., 63, 635

Nakariakov, V. M., \& Oraevsky, V. N. 1995, Solar Phys., 160, 289

Nakariakov, V. M., Roberts, B., \& Murawski, K. 1997, Solar Phys., 175, 93

Nakariakov, V. M., Roberts, B., \& Murawski, K. 1998, A\&A, 332,795

Nakariakov, V. M., Ofman, L., \& Arber, T. D. 2000, A\&A, 353,741

November, L. J., \& Koutchmy, S. 1996, ApJ, 466, 512

Ofman, L., Romoli, M., Poletto, G., Noci, G., \& Kohl, J. L. 1997, ApJ, 491, L111

Oliver, R., Ballester, J. L., Hood, A. W., \& Priest, E. R. 1993, A\&A, 273, 647

Parker, E. N. 1992, J. Geophys. Res., 97, 4311

Phillips, J. L., Balogh, A., Bame, S. J., et al. 1994, Geophys. Res. Lett., 21, 1105

Press, W. H., Flannery, B. P., Teukolsky, S. A., \& Vetterling, W. T. 1989, Numerical Recipes (Cambridge Univ. Press), pp. 423 and 430

Rae, I. C., \& Roberts, B. 1982, ApJ, 256, 761

Roberts, B. 1985, Phys. Fluids, 28, 3280

Roberts, B. 1991, in Advances in solar system magnetohydrodynamics, ed. E. R. Priest, \& A. W. Hood (Cambridge Univ. Press), 105

Stone, J. M., \& Norman, M. L. 1992, ApJ, 80, 753

Thomson, D. J., Maclennan, C. G., \& Lanzerotti, L. J. 1995, Nature, 376, 139

Zalesak, S. T. 1979, J. Comp. Phys., 31, 335

Zirker, J. B. 1993, Solar Phys., 148, 43 